\title{
Microvascular abnormalities in the mucosal prolapse syndrome
}

\author{
R N Lonsdale
}

Department of

Histopathology, Norfolk and Norwich Hospital, Norwich, Norfolk R N Lonsdale

Correspondence to:

Dr R N Lonsdale, Department of Histopathology, Norfolk \& Norwich Hospital, Brunswick Road, Norwich, Norfolk NR1 3SR.

Accepted for publication 8 June 1992

\begin{abstract}
Twenty two cases of colorectal mucosal prolapse from a variety of clinical situations were examined histologically to identify changes in the microvasculature which may predispose to ulceration. The findings were compared with 10 tubular adenomas and 10 hyperplastic polyps. Fibrin insudation into vessel walls and thickened mucosal vessels were significantly associated with the presence of ulceration. Possible mechanisms of ulceration are discussed, and an hypothesis based on a microvascular pathogenesis is presented. (Gut 1993; 34: 106-109)
\end{abstract}

In 1829 Cruveilhier described a case of nonneoplastic ulceration of the rectum. ${ }^{1}$ Since then there have been numerous reports of a similar condition which has become known as the 'solitary rectal ulcer syndrome' ${ }^{2-5}$ This disorder affects young adults and is characterised by rectal pain, altered bowel habit, and the passage of blood and mucus per rectum. Lesions typically comprise solitary or multiple superficial ulcers of the anterior or anterolateral rectal mucosa. A preulcerative phase is also recognised with hyperaemic, granular or polypoid mucosa. ${ }^{26}$ The condition is often associated with rectal prolapse, either overt or occult, ${ }^{3-7}$ and excessive straining

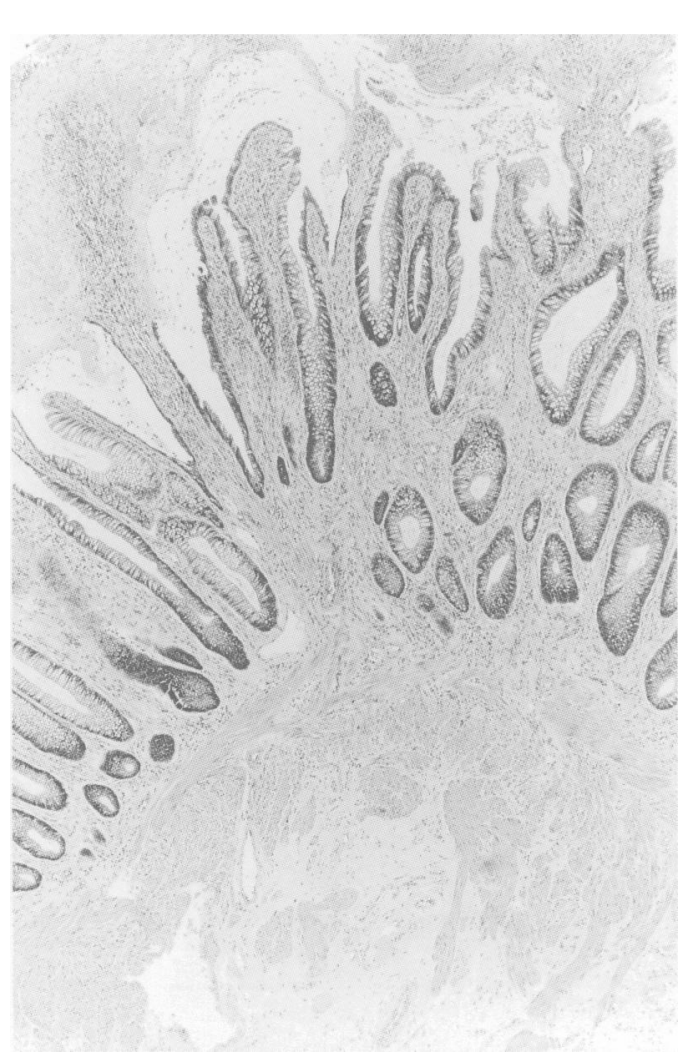

at stool. ${ }^{8}$ In some patients there is evidence of dysfunction of the defecation mechanism. ${ }^{79}$

Histologically the mucosa is abnormal with hyperplastic distorted crypts, fibrosis, and muscularisation of the lamina propria, thickening and splaying of the muscularis mucosae, and variable surface erosion (Fig 1). Resection specimens may show submucosal fibrosis and hypertrophy of the muscularis propria. The recognition that similar mucosal abnormalities occurred in other situations in the colorectum, such as adjacent to adenomas, carcinomas, prolapsed colostomies, 'colitis cystica profunda', descending perineal syndrome, ${ }^{10}$ polyps in Cowden's disease (multiple hamartoma syndrome)," and haemorrhoids, led to the 'unifying concept' of the mucosal prolapse syndrome by Du Boulay et al. ${ }^{12}$ The inflammatory cloacogenic polyp is thought to have a similar origin from prolapse of transitional mucosa at the anorectal junction. ${ }^{13}$

Although the histological features of mucosal prolapse syndrome are well recognised, very few authors have described abnormalities of blood vessels in this condition. In view of the potential importance of vascular changes in the pathogenesis of the ulceration that often accompanies mucosal prolapse syndrome, it was decided to study these changes in detail.

\section{Methods}

CASES

A search of the files of the Department of Histopathology, Norfolk and Norwich Hospital for the previous four years revealed 57 cases coded as mucosal prolapse syndrome. Of these, 22 cases had biopsies deep enough to assess the submucosal vasculature and were included in the study. Blocks were retrieved and sections stained with haematoxylin and eosin, Miller's elastin van Gieson, Martius scarlet blue, and Congo red. The specimens comprised nine cases of mucosal prolapse syndrome/solitary rectal ulcer, six cases of inflammatory cloacogenic polyp, four cases of overt rectal prolapse, one ileostomy ulcer, one colostomy polyp, and one nodule which developed at an anastomosis site. The clinical records of each patient were reviewed to identify patients with hypertension, diabetes mellitus, or amyloidosis. Ten consecutive tubular adenomas and 10 hyperplastic polyps were used as controls. Sections were screened paying particular attention to changes in the vessels of the mucosa and submucosa, and the presence of erosions or ulceration was noted.

Results

Demographic data and histological findings are sites of erosion. Crypts are hyperplastic, distorted
set in a fibrotic lamina propria. Note the thickened disorganised muscularis mucosae. (Haematoxylin and eosin). 
TABLE I Histological changes in mucosal vessels

\begin{tabular}{|c|c|c|c|c|c|c|c|c|c|c|}
\hline No & Age & Sex & Diagnosis & Erosion & Thrombosis & Ectasia & Musc & Fibrin & Endo & Atherosis \\
\hline 1 & 71 & $\mathrm{~F}$ & Prolapse & - & - & - & - & - & - & - \\
\hline 2 & 76 & $\mathbf{F}$ & Prolapse & - & - & + & - & - & + & - \\
\hline 3 & 50 & $\mathbf{F}$ & Prolapse & - & - & + & + & - & - & - \\
\hline 4 & 60 & $\mathbf{F}$ & Prolapse & - & - & + & - & - & - & - \\
\hline 5 & 41 & M & MPS & + & + & + & - & + & - & + \\
\hline 6 & 68 & $\mathrm{~F}$ & MPS & - & - & + & - & - & - & - \\
\hline 7 & 52 & $F$ & MPS & - & - & + & - & - & - & - \\
\hline 8 & 31 & M & MPS & - & - & + & - & - & - & - \\
\hline 9 & 33 & $\mathrm{~F}$ & MPS & - & - & + & + & - & - & - \\
\hline 10 & 73 & $\mathrm{~F}$ & MPS & + & - & + & + & + & - & + \\
\hline 11 & 17 & M & MPS & + & - & + & + & - & - & - \\
\hline 12 & 86 & $F$ & MPS & + & - & + & + & - & - & - \\
\hline 13 & 59 & $\mathrm{~F}$ & MPS & + & - & + & - & - & - & - \\
\hline 14 & 90 & $\mathrm{~F}$ & ICP & + & - & + & + & + & + & + \\
\hline 15 & 22 & $F$ & ICP & + & + & + & + & + & - & - \\
\hline 16 & 80 & M & ICP & + & - & + & + & - & - & - \\
\hline 17 & 69 & F & ICP & - & - & + & - & - & - & - \\
\hline 18 & 66 & M & ICP & + & + & + & + & + & - & - \\
\hline 19 & 77 & $M$ & ICP & + & - & + & + & - & + & - \\
\hline 20 & 78 & $\mathrm{~F}$ & AN & - & - & + & - & - & - & - \\
\hline 21 & 67 & F & IU & + & - & + & - & - & - & - \\
\hline 22 & 86 & M & $\mathrm{CP}$ & + & - & + & + & - & + & - \\
\hline \multicolumn{2}{|c|}{$\%$ of cases } & & & 55 & 14 & 95 & 50 & 23 & 18 & 14 \\
\hline
\end{tabular}

MPS=mucosal prolapse syndrome; $\mathrm{ICP}=$ inflammatory cloacogenic polyp; $\mathrm{AN}=$ anastomotic nodule; $\mathrm{IU}=\mathrm{ileostomy}$ ulcer; $\mathrm{CP}=$ colostomy polyp; Musc $=$ muscularised capillaries; Endo $=$ endothelial vacuolation .

summarised in Tables I and II. Five of the patients were hypertensive; one with overt prolapse, two with mucosal prolapse syndrome, one with an inflammatory cloacogenic polyp, and one with a colostomy polyp. None of the patients was diabetic or had amyloidosis.

All of the lesions had the typical architectural features of mucosal prolapse syndrome, with 12 of the 22 showing surface erosions. None of the biopsies included areas of full thickness mucosal ulceration. Mucosal capillary congestion and dilatation was an almost universal finding, and was most marked superficially. Vessels deeper in the mucosa showed muscularisation of their walls in half the cases (Fig 2). This finding was significantly associated with surface erosion. A frequent abnormality of mucosal capillaries and submucosal arterioles (predominantly the latter) was striking hyalinisation of the wall by insudation of fibrin. In some vessels this change appeared to occlude the lumen (Fig 3). There was no associated infiltrate of leucocytes to indicate a vasculitis. This reaction was also seen to a lesser extent in submucosal venules. Fibrin insudation was also significantly associated with surface erosion. Other changes noted less often were medial hypertrophy, intimal hyperplasia, and endothelial vacuolation of submucosal arteries. Three of seven examples of intimal hyperplasia, and four of eight examples of medial hypertrophy occurred in hypertensive patients. Lymphangiectasis was an inconstant finding, and thrombosis was rare, occurring only in capillaries beneath erosions. An interesting observation in five cases was the presence of atherosis - 'foamy' macrophages either within the wall or occluding the lumen of arterioles (Figs 3, 4). All five biopsy specimens with atherosis were eroded. Three of the 22 cases showed congophilic material within vessel walls. This did not, however, show the apple green birefringence characteristic of amyloid.

With the exception of dilated mucosal capillaries, these vascular abnormalities were not seen in either tubular adenomas or hyperplastic polyps.

TABLE II Histological changes in submucosal vessels

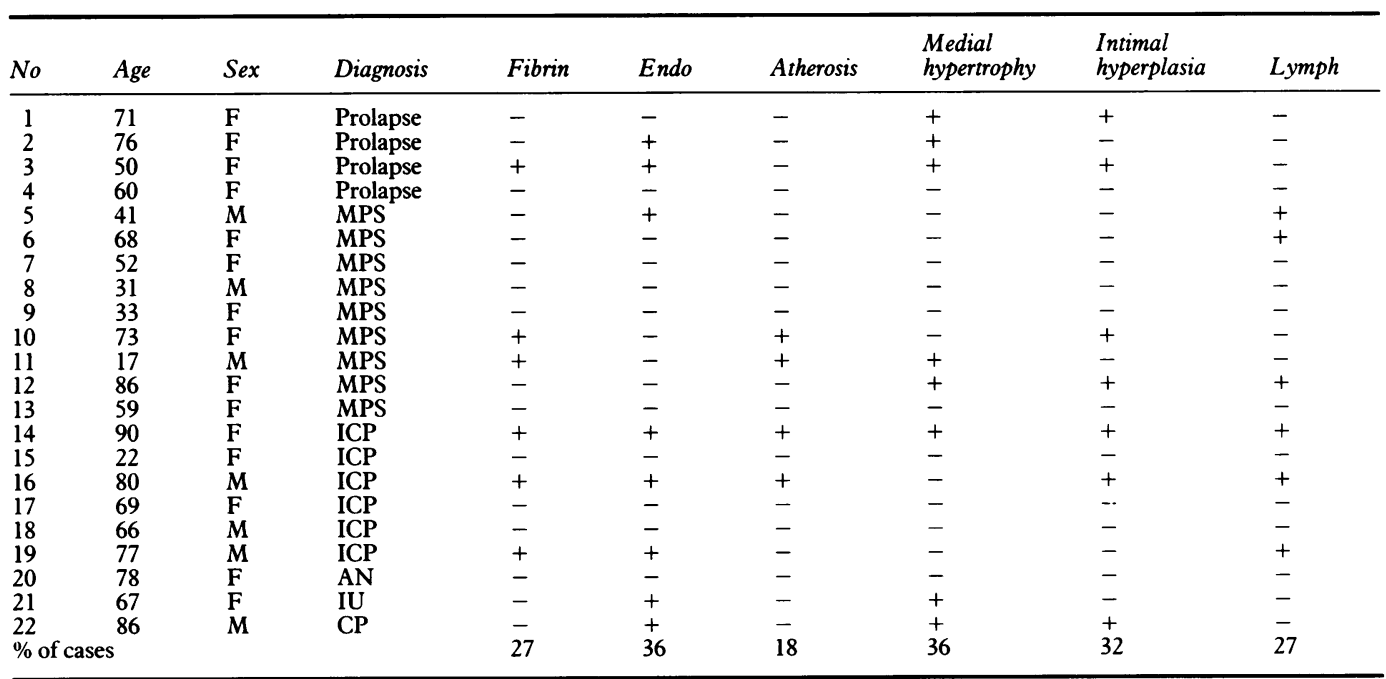

(For abbreviations see Table I). Capillary muscularisation and fibrin insudation showed a significant association with erosion (combined data $-\chi^{2} 4.58$ and 5.09 respectively, $D F=1, p<0.05$ with Yate's correction for small numbers). 
Figure 2: Thick walled muscularised muscosal blood vessel with vacuolated endothelial cells.

(Haematoxylin and eosin).

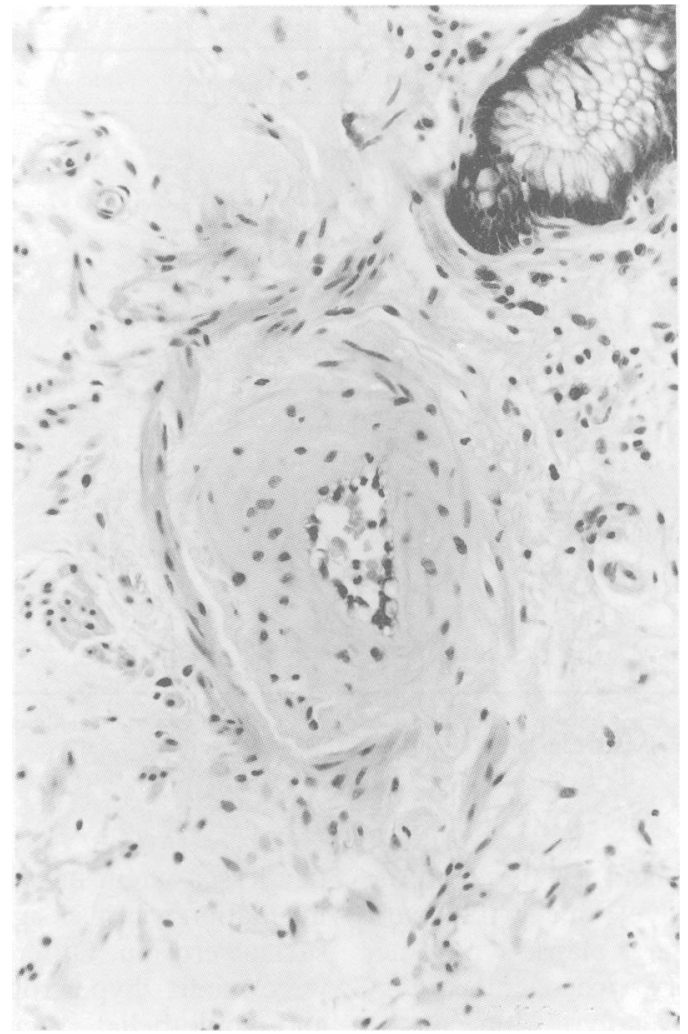

\section{Discussion}

The mechanism of ulceration in mucosal prolapse has been variously attributed to one or more of the following: ${ }^{8}(1)$ Pressure necrosis caused by mucosal impaction in the anal canal. (2) Ischaemia secondary to stretching and rupture of submucosal blood vessels. (3) Trauma during digital replacement of the prolapsing mucosa. (4) Ischaemia secondary to obliteration of mucosal capillaries by the fibromuscular sclerosis of the lamina propria.

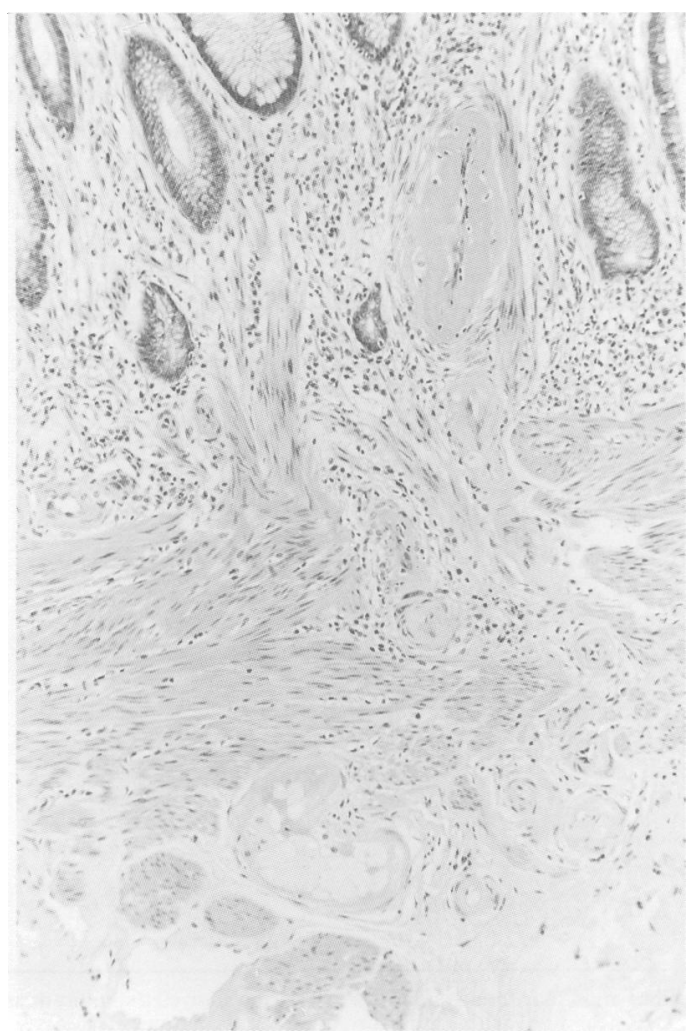

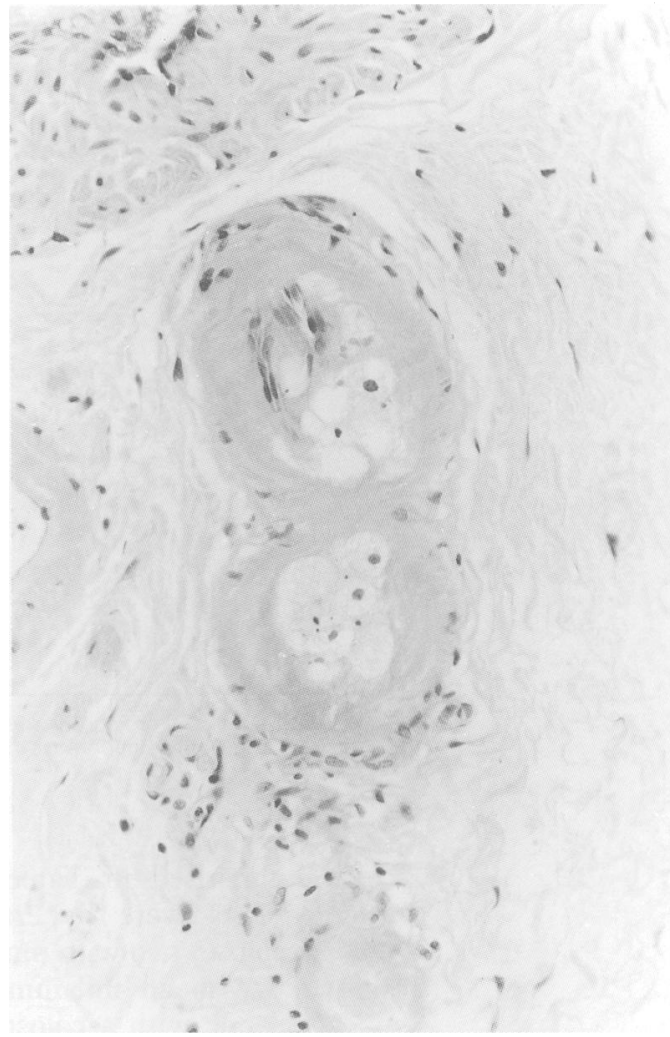

Figure 4: Atherosis - 'foamy' macrophages fill the lumen of a small arteriole. (Haematoxylin and eosin).

The latter two mechanisms probably have little role, if any, in most patients, and the results of this study reveal that far from being obliterated the mucosal capillaries are, in fact, dilated. Ischaemia is widely held to be important and yet, to the author's knowledge, there have been no systematic studies of vascular changes in this condition. The frequent finding of a spray of inflammatory exudate at sites of erosion (Fig 1), similar to that seen in pseudomembranous colitis (a condition in which ischaemia is also thought to play a role) adds further weight to an ischaemic pathogenesis. In this context the observation of fibrin insudation into vessel walls is of interest. This change is almost certainly identical to that described as 'fibrinoid necrosis' of arterial and arteriolar walls seen in three previous cases. ${ }^{812}$ Narrowing or occlusion of the lumen by this process provides a basis for ischaemic ulceration.

The accumulation of 'foamy' macrophages (atherosis) in vessel walls and lumina resembles that seen in early atheroma, pre-eclampsia, and after radiotherapy. Both atherosis and fibrin insudation imply damage to the endothelial lining, with leakage of plasma constituents into vessel walls. Endothelial vacuolation may be another manifestation of this damage. The following mechanism of ulceration is proposed: A prolapsed fold of mucosa submits submucosal and mucosal vascular endothelium to stretching and shearing forces. This damages the endothelium and causes leakage of plasma constituents into the wall, with subsequent narrowing of the lumen and distal ischaemia. Impaction of the prolapsed fold in the anal canal impairs venous drainage with resulting capillary congestion and slowing of the microcirculation. Thus both afferent and efferent vascular changes contribute capillary with obliteration the lumen (upper part of figure). A vessel beneath the thickened disorganised muscularis mucosae (lower part of figure) shows lumina occlusion by atherosis (Haematoxylin and eosin). 


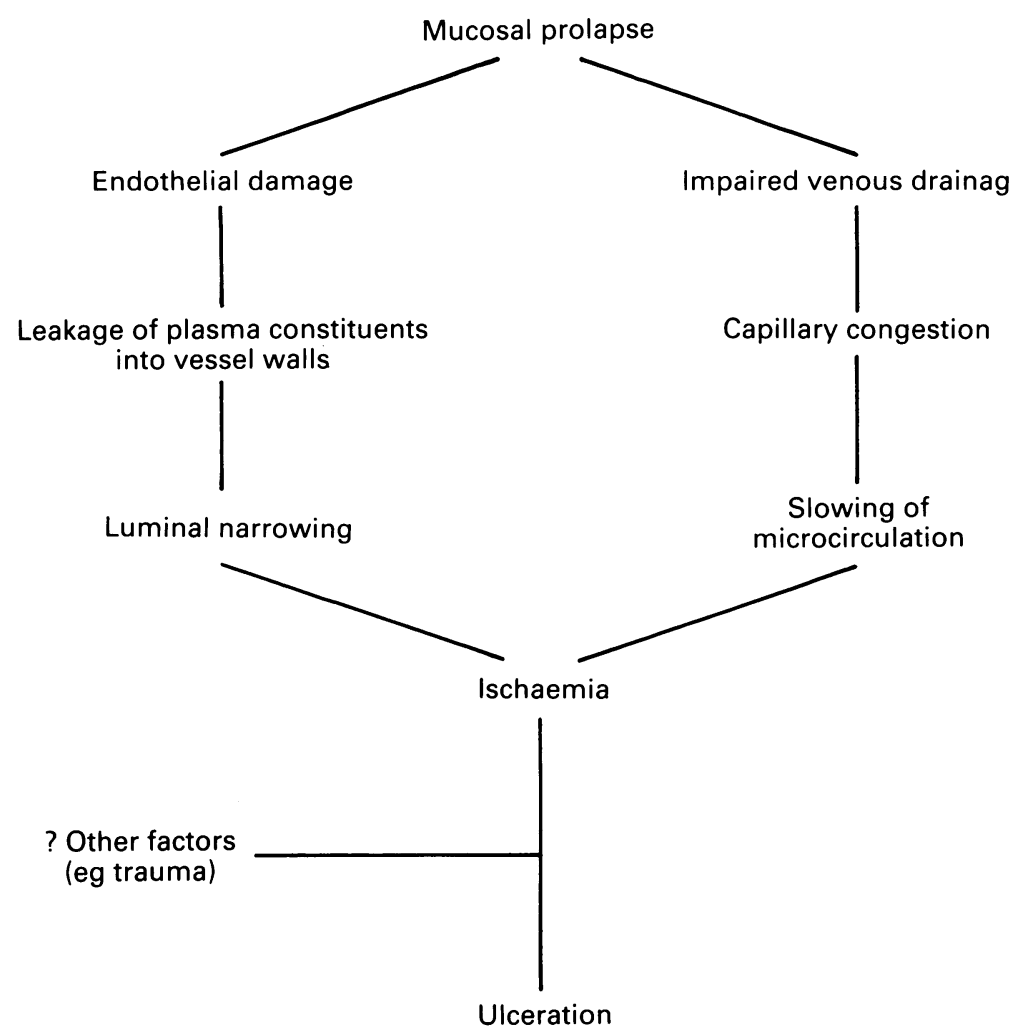

Figure 5: Proposed pathogenesis of ulceration in mucosal prolapse syndrome.

to ischaemia and subsequent ulceration. This hypothesis is shown diagrammatically in Figure 5. The thickened, muscularised capillaries seen in the base of the mucosa may be a response to local venous hypertension.

No vascular changes were observed in either the tubular adenomas or hyperplastic polyps apart from capillary ectasia. Hyperplastic polyps are usually small and sessile and unlikely to predispose to prolapse of adjacent mucosa. Many of the tubular adenomas were large and pedunculated, however, and although adjacent non-neoplastic mucosa occasionally showed features suggestive of prolapse, characteristic vascular changes were absent. This suggests that factors other than simple traction of mucosa into the lumen are responsible for the vascular changes observed in mucosal prolapse syndrome, and supports the hypothesis that efferent vessels are also important in the genesis of ulceration.

It is possible that the vascular abnormalities are secondary to the surface erosion rather than being causal. This may be the case with thrombosis of the superficial capillaries. Most of the abnormal vessels, however, are deep within the mucosa and submucosa, well away from the local influence of surface erosion, and most likely contribute to the mucosal loss rather than result from it.

Thanks are due to Dr R Y Ball for his constructive comments, and to Mrs S Brown for technical assistance.

1 Cruveilhier J. Ulcere chronique du rectum. In: Anatomie pathologique du corps humaine; maladies du rectum. Paris: I B Ballière, 1829: 25.

2 Madigan MR, Morson BC. Solitary ulcer of the rectum. Gut 1969; 10: 871-81.

3 Rutter KRP. Solitary rectal ulcer syndrome. Proc $R$ Soc Med 1975; 68: $22-6$

4 Schweiger M, Alexander-Williams J. Solitary-ulcer syndrome of the rectum. Its association with occult rectal prolapse. Lancet 1977; i: 170-1.

5 Ford MJ, Anderson JR, Gilmour HM, Holt S, Sircus W, Heading RC. Clinical spectrum of "solitary ulcer" of the rectum. Gastroenterology 1983; 84: 1533-40.

6 White CM, Findlay JM, Price JJ. The occult rectal prolapse syndrome. Br F Surg 1980; 67: 528-30.

7 Womack NR, Williams NS, Holmfield JHM, Morrison JFB Pressure and prolapse - the cause of solitary rectal ulceration. Gut 1987; 28: 1228-33.

8 Rutter KRP, Riddell RH. The solitary ulcer syndrome of the rectum. Clin Gastroenterol 1975; 4: 505-30.

Ruter KRP. Electromyographic changes in certain pelvic floor abnormalities. Proc $R$ Soc Med 1974; 67: 53-6.

10 Mackle EJ, Parks TG. The pathogenesis and pathophysiology of rectal prolapse and solitary rectal ulcer syndrome. Clin of rectal prolapse and solitary recta

11 Carlson GJ, Nivatvongs S, Snover DC. Colorectal polyps in Cowden's disease (multiple hamartoma syndrome). Am $\mathcal{F}$ Surg Pathol 1984; 8: 763-70.

12 Du Boulay CEH, Fairbrother J, Isaacson PG. Mucosal prolapse syndrome - a unifying concept for solitary rectal ulcer syndrome and related disorders. F Clin Pathol 1983; 36: $1264-8$.

13 Saul SH. Inflammatory cloacogenic polyp: relationship to solitary rectal ulcer syndrome/mucosal prolapse and other bowel disorders. Hum Pathol 1987; 18: 1120-5. 